\title{
Nuclear Cardiology - In the Era of the Interventional Cardiology
}

\author{
Branislav Baskot, Igor Ivanov, Dragan Kovacevic, \\ Slobodan Obradovic, Nenad Ratkovic and \\ Miodrag Zivkovic
}

Additional information is available at the end of the chapter

http://dx.doi.org/10.5772/55484

\section{Introduction}

The strength and breadth of nuclear cardiology lie in its great potential for future creative growth. This growth involves the development of new biologically derived radiopharmaceuticals, avdanced imaging techologies, and a broad/based set of research and clinical aplications involving diagnosis, functional categorization, prognosis, evaluation of therapeutic interventions, and the ability to deal with many of the major investigative issues in contemporary cardiology such as myocardial hibernation, stunning, and viability. The past decade has been caracteriyed by major advances in nuclear cardiology that have greatly enhanced the clinical utility of the various radionuclide techniques used for the assessment of regional myocardial perfusion and regional and global left ventricular function under resting and stress condotions. Despite the emergence of alternative noninvasive techniques for the diagnosis of coronary aretry disease (CAD) and the assessment of prognosis of viability, such as ergo- stress tests, stress echocardiography, the use and application of nuclear cardiology techniques have continued to increase. The establishment of the American Society of Nuclear Cardiology (ASNC) and its educational programs has led to a greater diffusion on nuclear cardiology technology in the community hospital setings and has promoted the emergence and dissemination of imaging and procedural guidelines for nuclear cardiology methods. The establishment of the Journal of Nuclear Cardiology, the official journal of ASNC, allowed a greater number of manuscript to be published in the field $[1,2,3]$

In the few past decade, significant advances have been made in the ability to image the heart with radionuclide tracers under stress and resting conditions in patientse with suspected or 
known coronary aretry disease (CAD) for the detection of ischemia, determination of prognosis, assessment of myocardial viability, preoperative risk assessment for patients undergoing noncardiac surgery, mand evaluation of the efficacy of revasculariyation in patients undergoing coronary artery bypass surgery or an interventional procedure $[1,2,3]$.

For many years, planar imaging and SPECT with 201Tl (201 Talium) constituted the only scintigrafic techniques available for detecting CAD and assessing prognosis in patients undergoing stress perfusion imaging. The major limitation of 201Tl scintigraphy is the high false/positive rate observed in many laboratories, wich is attributed predominantly to image attenuation aretfact and variants of normal that are interpreted as defects consequent to a significant coronary artery stenoses. Although quantification of 201Tl images improves specificity, the false/positive rate remains problematic, particulary in the women and in obese patients. Breast attenuation artifact in women are sometimes difficult to distiguish from perfusion abnormalities secondary to inducible ischemia or myocardial scar.

In recent years, new 99mTc (technetium) labeled perfusion agents have been introduced into clinical practice to enhance the specificity of Single Photon Emission Cumputed Tomography (SPECT) and to provide additional information regarding and global left ventricular systolic function via ECG gating of images $[3,4,8]$. It was immadiately apparent that the quality of images obtained with these $99 \mathrm{mTc}$-labeled radionuclides was superior to that images obtained with 201Tl because of the more favorable psysical characteristic of 99mTc imaging with gamma camera. Perhaps most importantly, 99mTc imaging allows easy gated acqusition, permiting simulateous evaluation of regional systolic thickening, global left ventricular function (LVEF), and myocardial perfusion. One the most significant avdances in myocardial perfusion imaging in the past decade is the development of quantitative SPECT perfusion imaging. Radionuclide imaging is an intrinsically digital technique that is ideally suited for quantification. A number of validated software packages are commercially available for quantification of SPECT myocardial perfusion and function (Auto Quant; Emory Toolbox; 4D/MSPECT; and Wackers Liu CQ), and are carried by the major vendors of nuclear medicine imaging equipment. The basic principles of SPECTR quantification are similar for each of these software packages. Each commercially available package also includes software for computation of LVEF and left ventricular volumes from ECG-gated SPECT images [7, 9, 10, 11].

\section{Indications for nuclear cardiology procedures}

\subsection{Suspected coronary artery disease}

CAD is still the single greatest cause of death of men and women in the world, despite a declining total death rate. Using USA data over 459.000 deaths were due to CAD - 1 of every deaths. There are aproximatelly 2.2 million hospital discharges with CAD as the diagnosis annually.

The reduction of the morbidity and mortality due to CAD is thus primary importance to physicians and patients. Stress myocardial perfusion imaging (MPI) has emerged as an 
important noninvasive means of evaluating patients with suspected CAD, with over than 10 millions studies performed in USA annually $[1,2,16,17,18]$.

\section{Risk factor assessment}

The first step in evaluating patients for CAD involves the assessment of the presence of traditional risk factors. Modifiable risks include hypercholesterolemia, hypertension, diabetes mellitus, obesity, tobacco use, and physical inactivity. Nonmodifiable risk factor includes a family history of CAD in first-degree relatives under the age of 60 , advanced age, and gender? Once risk factors associated with CAD are evaluated, a patient's risk for having CAD should be assessed. This is often performed by taking symptoms such as a chest pain, age, and gender into account. Symptoms suggestive of CAD, in addition to other risk factors, drive decisions for further testing $[2,12,17,18]$.

A cornerstone of the diagnosis of CAD has been exercise tolerance testing (ETT). The ETT is the safe and easily performed, usually in an office setting. But generally, ETT electrocardiography (ECG) has a sensitivity of 50 to $70 \%$, and a specificity of 60 to $80 \%$.

Thus, the major limitation of the ETT is its diagnostic accuracy for the detection of significant CAD. In patients able to exercise, the diagnostic accuracy of stress myocardial perfusion imaging (MPI) is significantly higher than the ETT alone and provides greater risk stratification for predicting the future cardiac events.

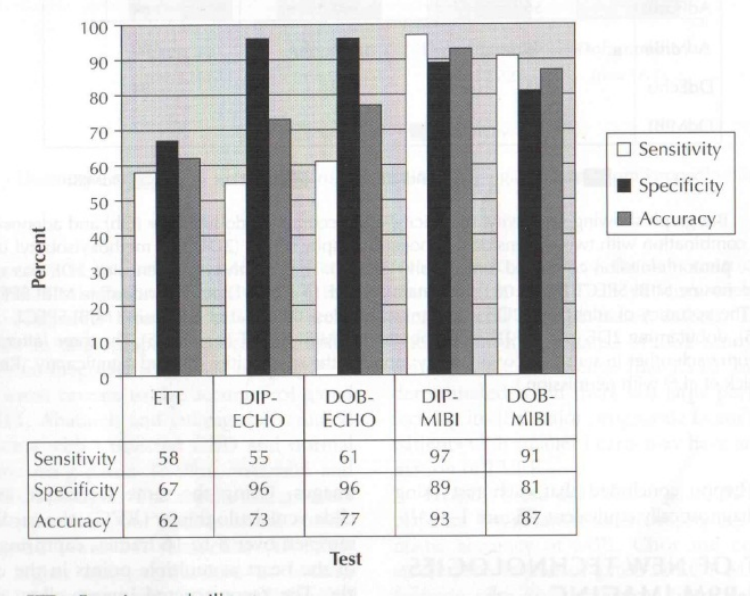

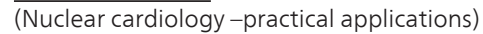

ETT exercise treadmill test

DIP-ECHO dipyridamole echocardiography

DOB-ECHO dobutamine echocardiography

DIP- MIBI dipyridamole myocardial perfusion imaging with Tc-99m MIBI

DOB-MIBI dobutamine myocardial perfusion imaging with Tc-99m MIBI
}

Figure 1. Diagnostic accuracy of various tests of CAD 
Because of limitation to performed exercise test (patients with medical illness, debilitation, musculoskeletal problems, and the older who can't reach a predicted maximum heart rate) MPI with pharmacologic stress using vasodilators (dipyridamole, and adenosine) or dobutamine can be implemented in such patients. In this moment, it has been estimated that $48 \%$ to $50 \%$ of all stress MPI is performed with pharmacologic agent. Briefly, dipyridamole and adenosine are potent coronary vasodilators that markedly increase coronary blood flow. This increased flow is less pronounced in arteries that are stenotic (flow restricted) due to atherosclerosis. This causes heterogeneous myocardial perfusion, which can be observed using that follows coronary blood flow as an alternative to vasodilator stress. Dobutamine works by increasing myocardial oxygen demand (through increased heart rate, systolic blood pressure, and myocardial contractility) $[5,6,7,8,9]$. As in exercise MPI scintigraphic images obtained at rest compared to those obtained during peak pharmacologic stress to distinguish myocardial ischemia from scar tissue (infarct area).

The diagnostic accuracy of Tc-99m imaging with pharmacologic stress test for angiographically significant CAD has been evaluated in numerous study.

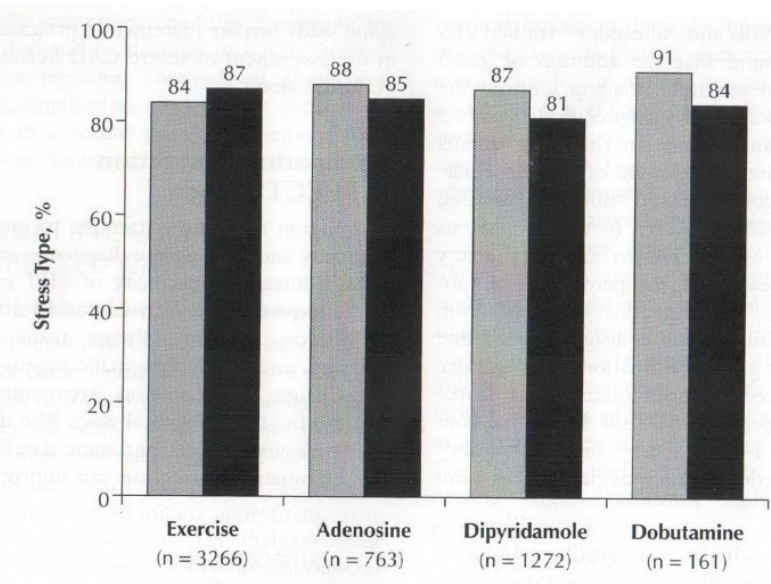

$\overline{\text { (Nuclear cardiology }}$-practical applications)

Figure 2. Diagnostic accuracy of stress myocardial perfusion imaging

\subsection{Evaluating and determination CULPRIT lesion, in indication for interventional cardiology}

One of the most powerfull uses of MPI is the evaluation of the risk for future events in patients with suspected or known CAD. Over the years, MPI has evolved as an essential tool in the evaluation and assessment of patient prior to coronary revascularization. It has a dual role. Prior to coronary angiography, MPI is extremly useful in documeting ischemia and determining the functional impact of single or multiple lesions indentified subsequently. After coronary 
anatomy is known, and despite some limitations in the setting of multivessel disease, MPI remains the test of choise for indentifying the lesion responsible for the ischemic symptoms, or so colled culprit lesion. That is extremly useful for futher management decisions with respect to percutaneous interventions. In compare, the absence of reversible ischemia in patients with known CAD is an excellent prognostic marker and predicts a low annual event rate.

The current definition of culprit lesion that is zone of ischemia under the coronary stenoses is not quite wright, because that is not definy two pathophysiologic aspects of ischemia; severity and extent. The primary objective of those study was to determinate and localizes culprit lesion by newly introduce parameters SRS (summary reversible score) and ISRS (index of summary reversible score), under the angiographically detected coronary narrowing $\geq 75 \%$ for the least one coronary artery $[2,6,9,11,15]$.

In the past two decades, a great body of literature has established the use of nuclear imaging for risk stratification in patients with known or suspected coronary artery disease (CAD). Risk stratification is of crucial importance for the practice of contemporary medicine. Extending the paradigm of noninvasive cardiac testing beyond the detection of disease is especially important, may risk assessment permits patients who are identified as being at a high risk for subsequent cardiac events should receive aggressive management, possibly including cardiac catheterization for potential revascularization procedures that may improve their outcome. CAD is disease with a wide spectrum of severity and extent with outcome, such as nonfatal myocardial infarction (MI) or cardiac death being related to the severity of disease. Clinical trials have shown that patients with severe CAD as left main coronary artery disease, especially those with left ventricular dysfunction, can benefit from coronary artery bypass graft surgery (CABG) with significant reduction in their mortality rate. Whereas patients with single-vessel or with two-vessel disease (without proximal left anterior descending artery involvement) would have improved symptoms of angina following CABG and percutaneous transluminal coronary angioplasty with or without stent implantation, without any effect on their mortality rate.

Coronary angiography, considered the "gold standard" for the diagnosis of CAD, often does not provide information about the physiologic significance of atherosclerotic lesions, especially in borderline lesions. More importantly, it does not provide a clear marker of risk of adverse events, especially in patients with moderate disease severity. Andreas Gruentzig said; "When coronary angiography founded coronary artery disease, I would like to have diagnostic procedure who will give me functional significance that lesion." $[2,10,12,18]$.

The presence of normal scintigraphic MPI study at a high level of stress ( $\geq 85 \%$ of maximum predicted heart rate) or proper pharmacologic stress carries a very benign prognosis, with mortality rate less than $0.5 \%$ per year. This finding has been reproduced in many studies. Iskander and Iskandiran, pooling the results of SPECT imaging from more than 12000 patients in 14 studies, demonstrated that the events rate (death/MI) for patients with normal MPI finding is $0.6 \%$, whereas abnormal study carries $7.4 \%$ per year event rate, a 12 -fold increase $[2,3,14,18]$. 
The current definition of culprit lesion; that is zone of ischemia under the coronary stenoses (what degree? That is not definition. Some autors ofer degree of stenoses $\leq 70 \%$, some $\leq 75 \%$, even $<80-85 \%$ ) is not quite wright, because that is not definy two pathophysiologic aspects of ischemia; severity and extent. Iskander and Iskadrian have also shown that defects reversibility is an important predictor of type of cardiac events, whereas reversible perfusion defects are associated with nonfatal MI. This is very important finding, since a reversible defect on MPI imaging is the only available diagnostic tool that can independently predict the risk of nonfatal MI. Therefore, stress perfusion studies should be reported documenting defect severity (mild, moderate, severe), size (small, moderate, large) and reversibility to provide essential risk stratification.[2, 3, 16].

The size and severity of the perfusion abnormality provide powerful prognostic information and has been shown to directly relate to outcome. MPI perfusion imaging and determination of culprit lesion is more predicitble of cardiac events than coronary angiography. As MPI imaging may identify those patients at high risk for subsequent cardiac events, perfusion imaging may be used to help guide further testing and revascularization procedures, and this obviously has important cost-effectiveness ramifications.

The primary objective of this study was to determinate and localizes culprit lesion by newly introduce parameters SRS (summary reversible score) and ISRS (index of summary reversible score), under the angiographically detected coronary narrowing $\geq 75 \%$ for the least one coronary artery [2].

The rapid rates of technical advances and improved operator expertise have enabled this technique to gain more widespread application. Despite the large number of PTCA-s performed yearly, preprocedure documentation of myocardial ischemia is uncommon, occurring in only $29 \%$ of patients.

Myocardial perfusion imaging provides information on the extent and location of myocardial ischemia. The assessment of jeopardized myocardium may be performed and provides a measure of the relative value of PTCA in terms of the amount of jeopardized myocardium. The location of the stenosis may dictate the area at risk: extent and severity of perfusion defects were significantly smaller in patients with proximal compared with distal coronary artery occlusions.

Before revascularization is performed, myocardial perfusion imaging may assist in management decisions by demonstrating the presence of myocardial ischemia, viability and delineating the severity and extent of coronary artery disease. The significance of equivocal lesions may be determined and culprit vessel may be successfully defined by SPECT imaging before angioplasty $[2,3,10,18]$.

The coronary angiography provides information on the anatomical state of the coronary tree and, specifically, on the large epicardial arteries, while perfusion SPECT facilitates the evaluation of the grade of ischemia that a particular stenosis produces. MPI SPECT is of considerable use in the procedural indications of partial revascularization in patients with chronic coronary artery disease (CAD). In these cases the purpose is to detect the coronary stenosis that provokes the ischemia and is termed the "culprit lesion". 
The aim of the study Baskot at all. [2] was to determine and localize culprit lesion by MPI in cases of angiographically detected coronary narrowing $\geq 75 \%$ of at least one coronary artery.

In the study four hundred and thirty-seven [437] patients were studied. In all of them angiographically detected significant coronary narrowing ( $\geq 75 \%$ luminal stenosis) before PCI. All the patients were submitted to MPI ${ }^{99 \mathrm{~m}} \mathrm{Tc}-\mathrm{MIBI}$, with pharmacologic dipyridamole stress protocol with concomitant low level bicycle exercise $50 \mathrm{~W}$ (DipyEX). We measured relative uptake ${ }^{99 \mathrm{~m}}$ Tc-MIBI for each myocardial segment using short-axis tomogram study. A 5-point scoring system was used to assess the difference between uptake degree in stress and rest studies for the same segment, and we created two indices: Sum reversible score (SRS), Index of sum reversibility score (ISRS). In the results a total 1311 vascular territories (7429 segments) were analyzed before elective percutaneous coronary intervention (ePCI). Overall sensitivity, specificity and accuracy using SRS were $89.7 \%, 86,7 \%$, and $88,2 \%$, with a positive predictive value of $92,7 \%$. Overall sensitivity, specificity and accuracy using ISRS were $92.8 \%, 89.1 \%$, and $92.3 \%$, and the positive predictive value was $93.7 \%$. Conclusion this work that is DipyEX MPI with two indices created SRS and ISRS significantly improves sensitivity, specificity and accuracy in the determination and localization of culprit lesion in patients undergoing elective PCI. In this work author defined culprit lesion using two physiological aspects; severity of ischemia and extension zone of ischemia. With quantification of these two parameters of culprit lesion, the author determined patients who underwent ePCI with stent implantation, and who had the best therapy effects with PCI therapy.

\section{Case 1.}

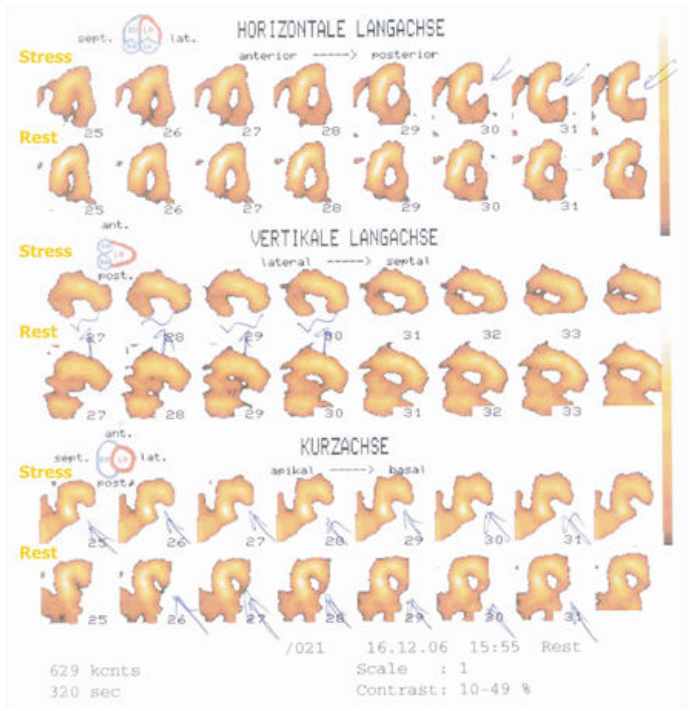

Figure showed culprit lesion in the inferolateral segments in the AdenoEx (up line slices) MPI study 

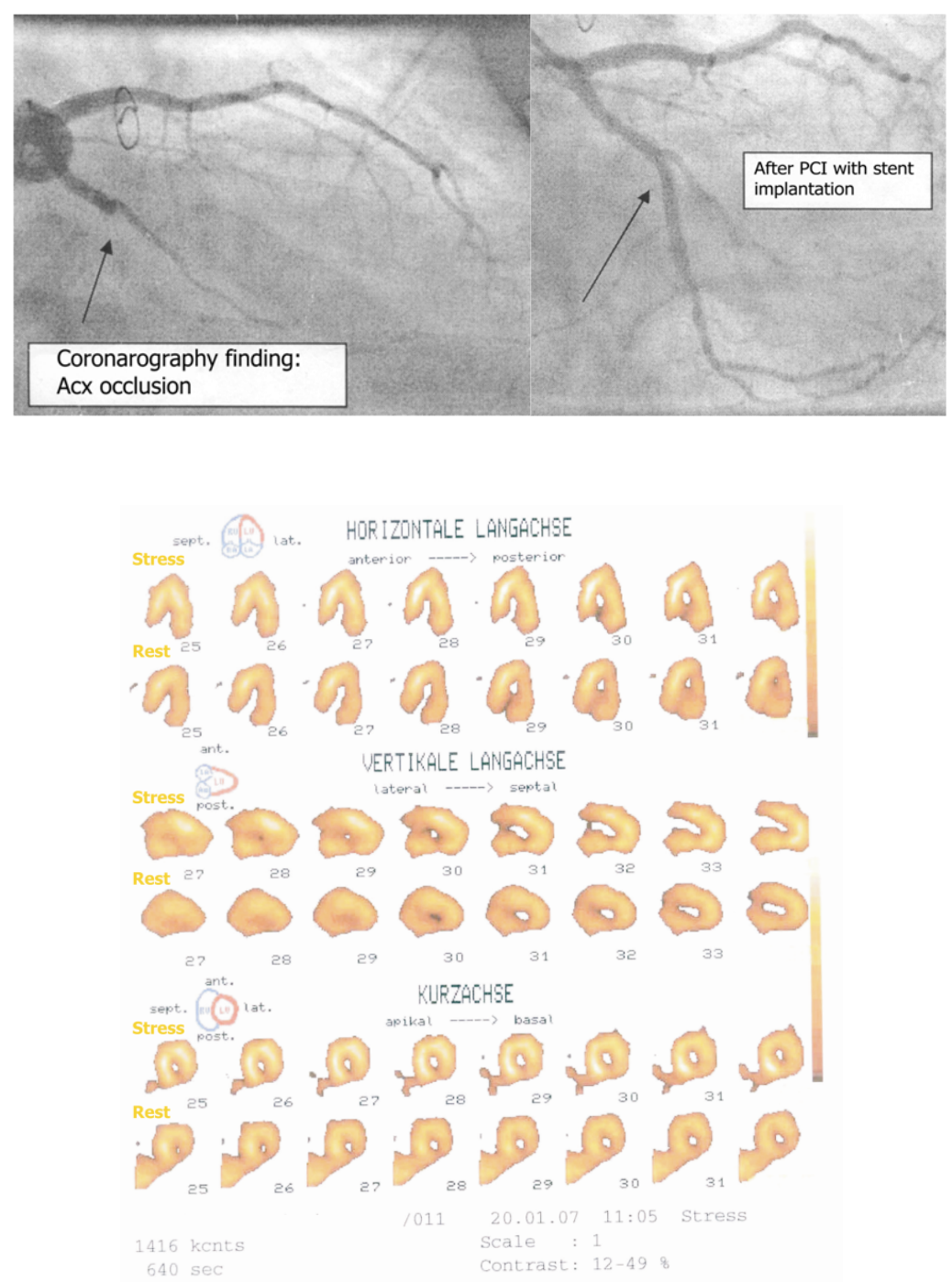

Nearly after elective PCI intervention we performed MPI with normal finding of perfusion

\section{Case 2.}

- Patient male 61 year old. St post IM with revascularization 1996 triple ACB (LIMA - LAD; venous graft on the D1 and

- In April 2010 performed SPECT MPI, finding sugested invasive intervention.

- Coronarography finding ;LM 90\%, LAD occluded, LIMA graft wide open. Venous graft on the D1 occluded. 
- ACx stenoses $90 \%$, OM with tubular stenosis $50-70 \%$

- RCA dominant, occluded ostial, venous graft occluded okludiran.

- Performed PCI with stent implantation (Tsunami gold $3.5 \times 15$ ) on LM and ACX

- After four month MPI control when we founded in stent stenosis.

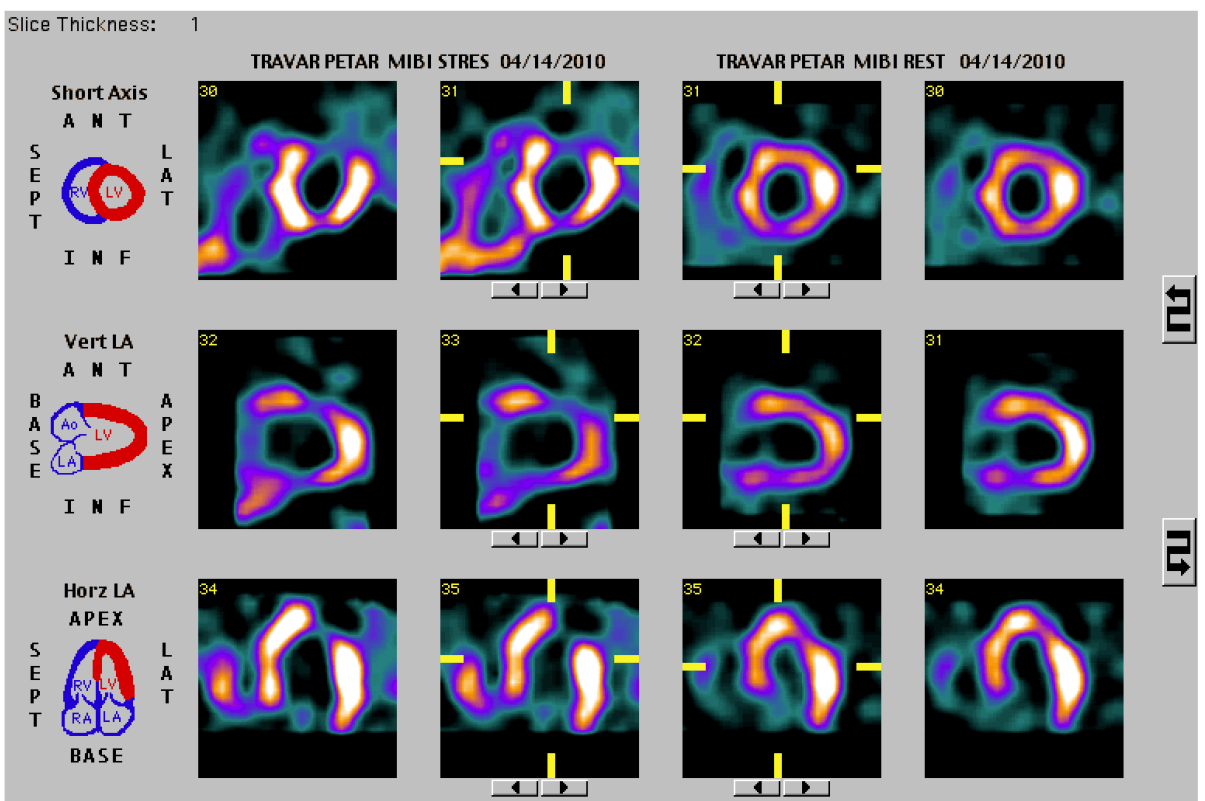

Culprit lesion
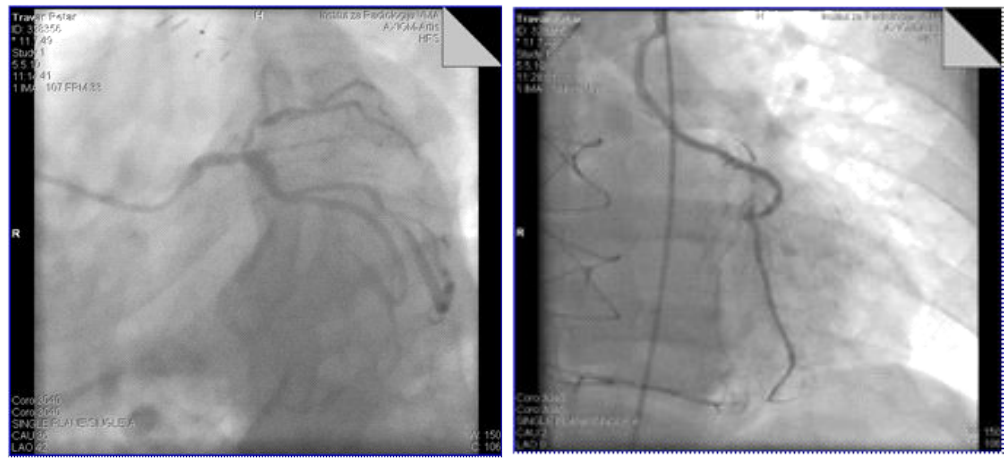

Coronarography finding 


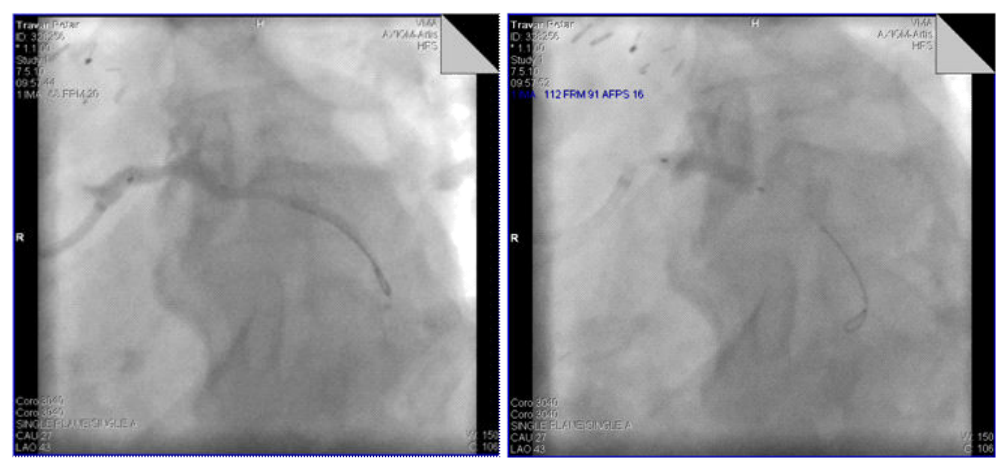

In the same act $\mathrm{PCl}$ with stent implantation

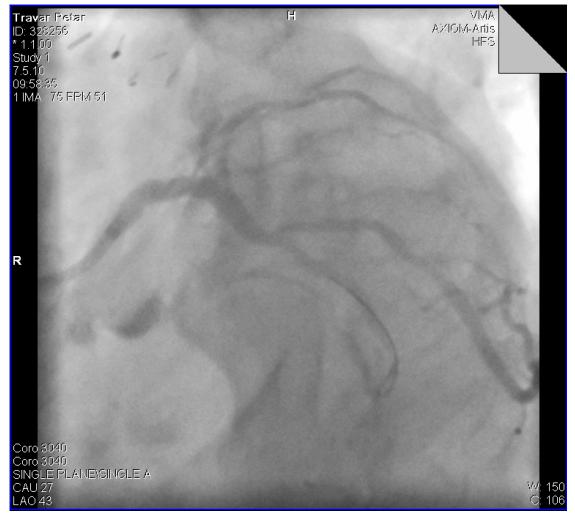

Final effect PCI 


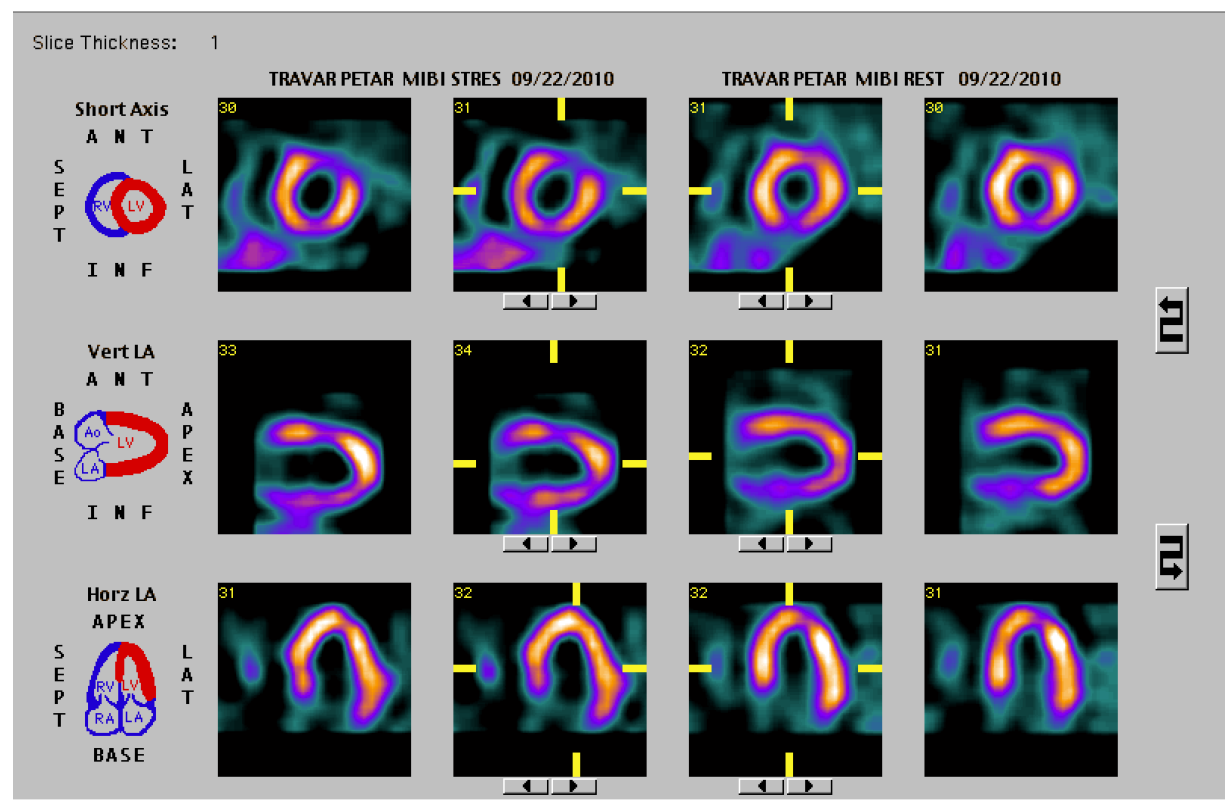

Control MPI after four month after PCI

We finding zone of reversible ischemia in the same area, suggest restenosis

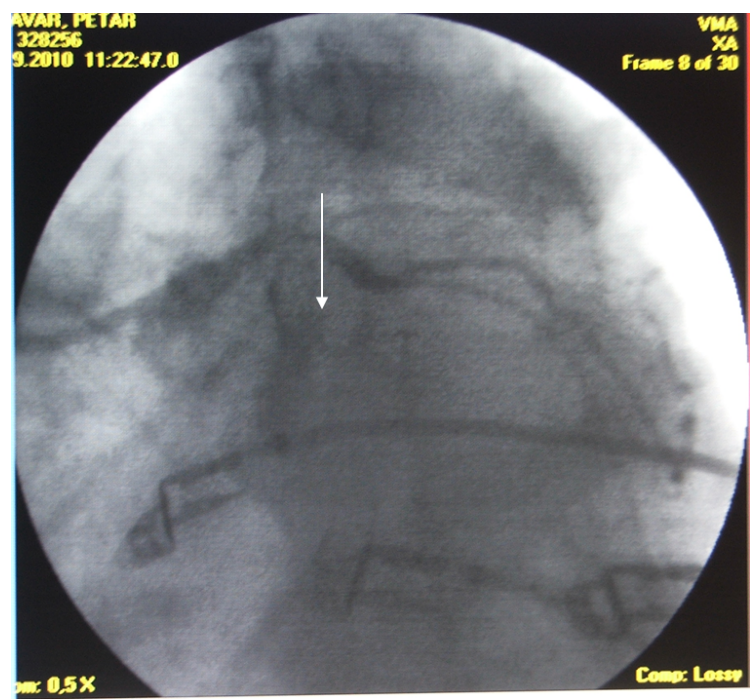

Control Coronarography - fidning in stent stenonis 

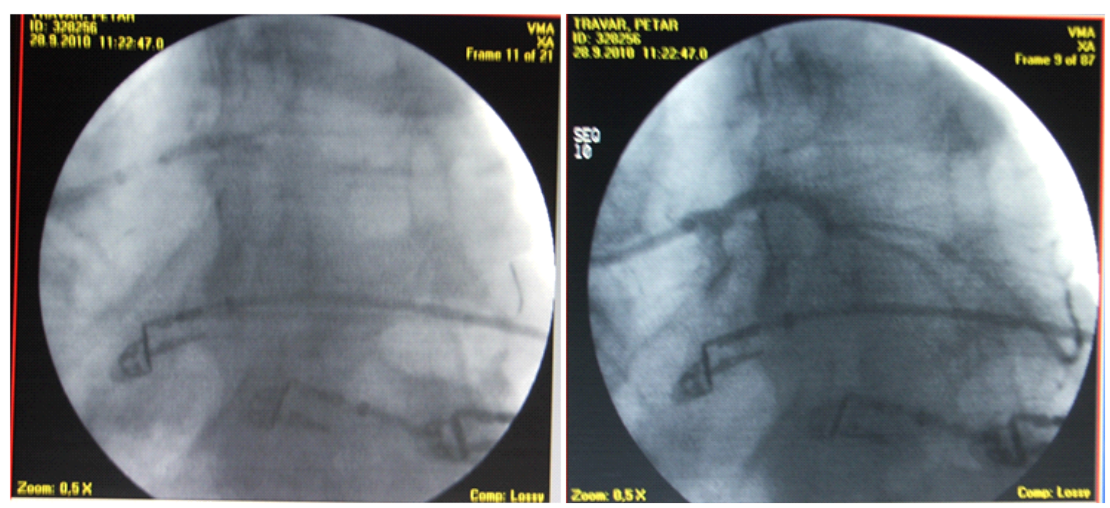

Re PCI with stent implantation
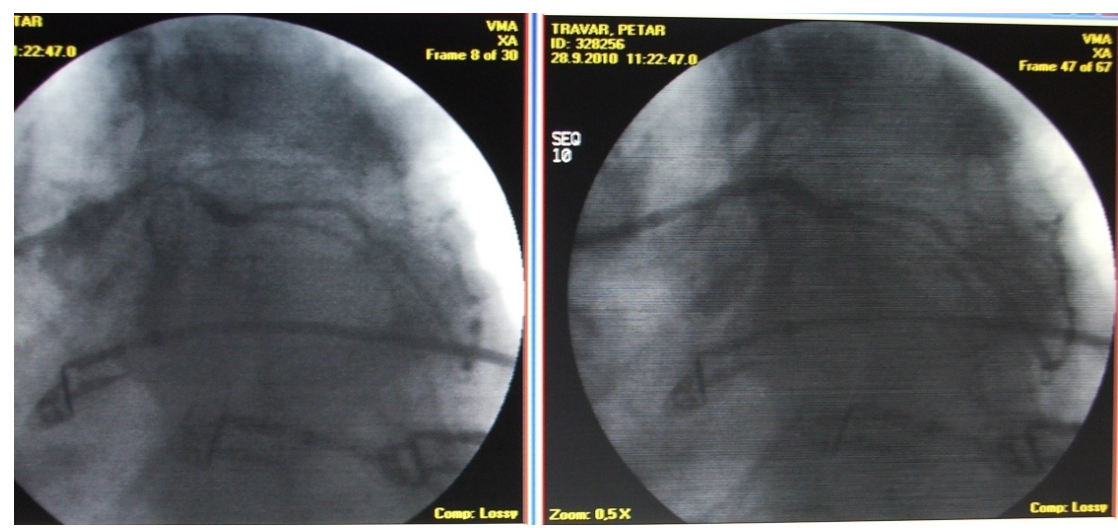

Final effect

Conversely, patients with high-risk scans may benefit from an early invasive strategy with a view toward revascularization depending on coronary anatomical finding. A substantial number of patients undergoing SPECT perfusion imaging will have mild ischemia without a multi-vessel disease scan pattern. If patients with mild ischemia have good exercise tolerance, they should be considered as candidates for intense medical therapy with follow-up exercise SPECT imaging possibly at 1 year. Unpublished data from the Clinical Outcomes Utilizing Revascularization and Aggressive Drug Evaluations (COURAGE) trial seem to indicate that many ischemic defects may markedly improve with aggressive lowering of abnormal lipids an the other pharmacological interventions. Hachamovitch and colleagues reported patients with the mildly abnormal scan had a $0.8 \%$ annual cardiac death rate compared with $0.9 \%$ for those who underwent revascularization. The death rate in medically treated patients who had moderately abnormal scans was $2.3 \%$ versus $1.1 \%$ for such patients undergoing revasculari- 
zation. Finally, patients with a severely abnormal scan treated medically had an annual cardiac death rate of $4.6 \%$ versus $1.3 \%$ for such patients who were revascularized. In the second study, these investigators showed that medically treated patients who had greater than $20 \%$ of the total myocardium rendered ischemic had higher annual cardiac death rate $(6.7 \%)$ compared with $2.0 \%$ for patients with this degree of extensive ischemia who underwent revascularization. For patients with $10 \%$ or less of the total myocardium rendered ischemic, there was no difference in outcome between medical therapy and revascularization.

Exercise myocardial perfusion imaging is a valuable adjunct for separating high to low risk patients who present symptoms consistent with stable CAD, or in patients who have known disease and in whom further prognostication is warranted. Multiple high-risk nuclear imaging variables can be identified, and the greater the extent of exercise/induced ischemia, the greater the risk of cardiac events. Adjunctive variables, such as transient ischemic cavity dilatation and functional assessment with evaluation of regional wall thickening or wall motion and left ventricular ejection fraction greatly assist in the risk stratification process $[1,3,16,18]$.

Nuclear cardiology is uniquely placed to address all the major determinants of prognosis in CAD can be assessed by measurements of stress-induced perfusion or function. These measurements include the amount of infarcted myocardium, the amount of jeopardized myocardium (supplied by vessels with hemodynamically significant stenosis), and the degree of jeopardy (tightness of the individual coronary stenosis). Recent evidence in large patient cohorts has revealed that factor estimating the extent of left ventricular dysfunction (left ventricular ejection fraction, extent of infarcted myocardium, transient ischemic dilatation of the left ventricle and increasing lung uptake) are excellent predictors of cardiac mortality. However, measurements of inducible ischemia are the best predictors of the development of acute coronary syndromes. Several reports have shown that nuclear testing yields incremental prognostic value over clinical information with respect to cardiac death, or the combination of cardiac death and nonfatal myocardial infarction as isolated endpoints. Now it is possible to tailor therapeutic decision making for an individual patient based upon combination of clinical factors and nuclear scan results. Patients with severe perfusion abnormalities on their stress image may have a five- to ten-fold higher likelihood of cardiac death versus patient with a normal myocardial perfusion SPECT. If the defects perfusion determined as a culprit lesion, invasive therapy (PCI) is an optimized outcome for that patient $[2,12,13,15]$.

The explosion of PTCA and stent placement in patients with single or multi-vessel disease has created a necessity for early detection of restenosis. A number of clinical studies have documented the usefulness of stress MPI for identifying restenosis in patients after PCI. One point of controversy is the optimal time to performing SPECT imaging after coronary intervention. Although current consensus in to obtain an exercise MPI study 4 to 6 weeks post intervention, whenever indicated, the proper timing for use of MPI remains to be determined. Based on existing knowledge about the timing interval of subacute thrombosis ( $<4$ weeks) and in-stent restenosis (3-6 month), we purpose the algorithm as a guide for the management of patients with known CAD after PCI [2, 15, 16, 18]. 
PTCA- Stent (PCI)

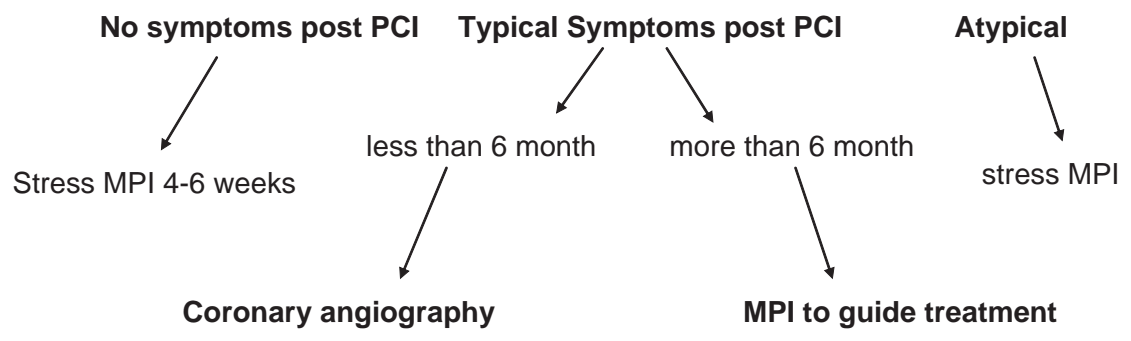

Figure 3. Recommendation for the diagnostic treatment after $\mathrm{PCI}$.

Asymptomatic patients may be considered for stress MPI 4 to 6 weeks post intervention in order to assess the functional results of PCI and established a new baseline. Subsets of patients that benefit from this approach include those at high risk post PCI (patients with decreased LV function, proximal left anterior descending artery disease, previous sudden death, diabetes mellitus, hazardous occupations, and suboptimal PCI results). Stress MPI is also recommended in patients who develop atypical symptoms after PCI and there is necessity to assess whether these symptoms represent ischemia. Patient with symptoms typical of ischemia $<6$ months post intervention should proceed with coronary angiography as a first step, unless contraindicated. If angina occurs later ( $>6$ months post PCI), stress/pharmacologic MPI can be used to assess degree and area of ischemia, since progression of native coronary disease rather than in-stent restenosis is more likely.

Incremental prognostic value of MPI; Because nuclear test are expensive the cost-effectiveness of these tests should demonstrate incremental prognostic information that cannot be derived from less expensive modalities such as clinical patient history and risks factors, standard ECG, and exercise ECG.

The combination clinical and exercise MPI variables provided greater prognostic information than the combination of clinical and angiographic data. Iskandrian et al showed that in medically treated patients with CAD, exercise SPECT imaging provided independent and incremental prognostic information even when catheterization data are available. The extent of perfusion abnormality was the single best predictor of prognosis. MPI added incremental prognostic information and risk-stratified patient even after clinical and exercise information were known. The incremental prognostic information about prognosis and need for coronary angiography provided by MPI has additionally been demonstrated for specific patients subsets: women, patient following coronary angioplasty or CABG, after MI, and with unstable angina. Hachamovitch et al demonstrated the use of MPI to yield incremental prognostic information toward the identification of cardiac death. Patient with a mildly abnormal scan after exercise stress are at low risk for cardiac death, but intermediate risk for nonfatal MI. A noninvasive strategy of optimizing medical therapy in this patient subgroup may result in significant cost saving when compared with invasive management strategy $[1,2,3,12,18]$. 
The prognostic efficacy of MPI is well established. Subsequent data have demonstrated in various patient subsets that nuclear tests add significant and incremental predictive value to less expensive clinical and exercise testing data. Angiographic data obtained from more expensive cardiac catheterization procedures add little or no significant incremental prognostic value when added to the results of MPI.

The introduction of new drugs and interventional devices to treat $\mathrm{CAD}$, coupled with the arrival of manage care, has led to era of cost-containment within the practice of cardiology. Stress MPI is increasingly seen as a gatekeeper for more costly diagnosis and interventional procedures. Steingart et al evaluated 378 patients with a full range of pretest probabilities for CAD, and demonstrated that the results of MPI significantly reduced referring physicians' likelihood of recommending cardiac catheterization, on average by $49 \%$.

Under managed care health systems that operate under cost-containment and capitation, MPI will continue to impact significantly on the decision to perform cardiac catheterization and to refer patients for coronary revascurization. Recommendations for invasive and interventional procedures are often coupled with an appropriate understanding of the prognostic value of MPI. Patients with the normal stress radionuclide study do not generally require referral for additional procedures, even when the likelihood of underlying CAD is high, as based on clinical and stress ECG data. The need for cardiac catheterization and coronary revascularization rates should be based on the degree of abnormality as detected by MPI. Thus, there is an increasing role of MPI to play an important gatekeeper function in the current era of managed care and emphasis on cost-containment $[2,3,8,12,18]$.

Stress MPI has became a central guide in decision making with regard to CAD patients. Stress MPI is commonly used either before consideration of coronary revascularization or after its performance, to optimize decision making for CAD patients. Stress MPI is also used after myocardial revascularization procedures, to evaluate therapeutic efficacy; following the stabilization of acute ischemic syndromes; to determine subsequent risk; and before the performance of elective non-cardiac surgery, to identify the high-risk subsets of CAD patients who will require coronary revascularization prior to elective surgery.

After all, MPI has became an important instrument in defining cardiac risk and in identifying patients who are most likely to benefit from additional invasive diagnostic testing and potential coronary revascularization. MPI demonstrated significant incremental prognostic

\section{Heart failure - New approach of therapy and diagnostic evaluation of therapy effect ${ }^{1}$}

Heart failure (HF) is becoming the main clinical challenge in cardiology in the twenty - first century and is associated with high morbidity and mortality. Heart failure is the third most

1 Zivkovic Miodrag, Baskot Branislav 
prevalent cardiovascular disease in the United States. An estimated 5 million people in the USA have heart failure, and the prevalence of the condition increase to 10 million by 2040, according to prediction. The prevalence of heart failure increase with age from less than $1 \%$ in the 20 - 30-year-old age group to over $20 \%$ in people age 80 years and older. The diagnostic and therapeutic costs involved are estimated to have exceeded $\$ 34$ billion in only one year. Despite advances in therapies, the long-term prognosis from patients with heart failure remain poor; $80 \%$ of men and $70 \%$ of women greater than 65 years of age with heart failure die within 8 years $[1,2,19,20,21]$.

\subsection{What we need for good and quality therapy}

The underllying etiology of HF needs to be determined; most patients have CAD (approximately $70 \%-80 \%$ ). Nuclear imaging can help in the differentiation between patients with ischemic and non-ischemic HF. In patients with ischemic cardiomiopathy, the precise coronary anatomy is also needed to determinate if revascularization needs to be considered. At present, invasive angiography is performed to obtain the coronary anatomy, but multi-slice CT (MSCT) may also provide this information. The presence of ischemia and viability needs to be determined to decide further if revascularization is indicated.

Nuclear imaging is considered the first choice technique for assessment of ischemia and viability; booth single-photon emission CT (SPECT), and positron emission tomography (PET) can provide this information.

Nuclear imaging can provide some indirect evidence in the differentiation between ischemic and non-ischemic HF. With stress,-rest SPECT study, reversible defects indicate ischemia and fixed defects of perfusion indicates scar tissue; booth this findings are markers of coronary artery disease. Moreover, lot of studies with nuclear perfusion imaging demonstrated that patients with ischemic HF had extensive and diffuse perfusion defects, whereas tracer uptake (myocardial perfusion) was mostly homogeneous (ischemic) in patients with non-ischemic HF.

Similarly, PET studies also demonstrated that patients with non-ischemic HF had more homogeneous tracer uptake, whereas patients with ischemic HF had areas of severely reduced uptake (reflecting scar formation). Accordingly, nuclear imaging can help in the differentiation between ischemic and non-ischemic cardiomyopathy, but for the diagnosis of underlying coronary artery disease, visualization of the coronary artery is needed. Invasive angiography is the technique of choice, but recently MSCT has been introduced for noninvasive angiography. With 64-slice MSCT and dual-source slice MSCT, we obtained more consistent image quality with improve visualization of the coronary artery tree. In the presence of a flow-limiting stenosis, resting myocardial is preserved, but once an increased myocardial oxygen demand occurs, a perfusion demand-supply mismatch follows, resulting in myocardial ischemia. Then, a sequence of events is initiated, which is referred to as the "ischemic cascade". Perfusion abnormalities occur at an early stage, whereas diastolic and systolic left ventricular dysfunction occur later. Accordingly, such 
techniques as nuclear imaging that defect perfusion abnormalities should have a high sensitivity for detection of ischemia, because these abnormalities occur early in the cascade $[19,20,21]$.

In the heart failure patients, the combination between the coronary anatomy and the presence of ischemia in the territories of the stenotic vessels determines the need for revascularization. In the absence of ischemia, the presence of viability needs to evaluation.

Another nuclear study performed in the evaluating of therapy effects in HF, is radionuclide angiography (RNV). That is the most reproducible, accurate, and simple method for noninvasively assessing left ventricular ejection fraction (LVEF). RNV are now most often used for serial assessment of LVEF in patients who undergo chemotherapy, assessment of global regional wall motion in patients with recent or old myocardial infarction, and in patients with congestive heart failure. In the patients with heart failure, evaluation of left ventricular systolic function is essential to plan management and determine prognosis. At the present time most RNV are acquired by multiple - view planar image technique. In this moment SPECT RNV is not routinely performed in most nuclear cardiology laboratories. The greatest attraction of SPECT RNV is the ability to evaluate cardiac chambers and regional wall motion without overlap of other structures. LVEF may be calculated based on count changes from either a conventional planar image (best septal) or from SPECT RNV.

In the pilot study Zivkovic, Baskot at all. We introduced new therapy, hyperbaric oxygenation (HBO) and erythropoietin (EPO) in the treatment of heart failure. The aim of this study is to show positive therapeutic effects of synergistic applications of $\mathrm{HBO}$ as a strong generator of regenerator activities in human tissues, and recombinant EPO as a general growth factor, in the treatment HF ischemic and non-ischemic origin [19, 20,21].

$\mathrm{HBO}$ is medical procedure of breathing the $100 \%$ oxygen under pressures higher than atmospheric pressure is and it carries out into hyperbaric chambers. Contemporary, HBO changes the rheological blood characteristic, recovers the function of blood vessels endothelium and it has good antiaggregation effects. The oxygen's pharmacokinetic and neo-angiogenesis effects and its effect on oxygen-dependent reactions inside the mitochondria's and homeopathy effect on the other organs are the reasons to use HBO in the treatment of HF. Nuclear imaging by SPECT imaging in the evaluation between ischemic and non-ischemic HF we performed, and followed with quantification per segments before and after therapy in the evaluation positive therapeutic effect. We also performed RNV like gold standard in the evaluation global and regional LVEF before and after the therapy. In the pilot study with 18 patients, we had recovery perfusion in all patients with nonischemic HF. Before therapy we finding with RNV average measured LVEF was $23.4 \%$. After treatment LVEF measured average 34, 3\%. The results was increase by 10, 9\% (from $5 \%$ to $20 \%$ measured individually).

Conclusion of this pilot study was that diagnostic information finding by nuclear cardiology (perfusion and function) suggested significantly positive therapy effects HBO and Erythropoietin in the therapy of heart failure ischemic and non-ischemic origin. 


\section{Case report 1. Patient female with multi vessel disease by coronarography}

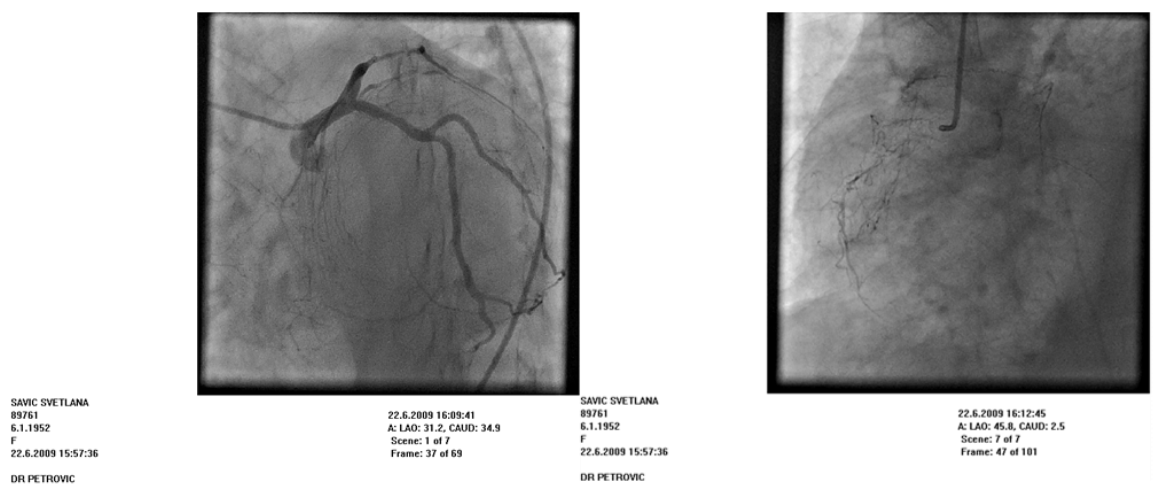

SPECT with DipyEX (dipyridamole = concomitant low level exercise 50W) performed and evaluated before and after $\mathrm{HBO}+\mathrm{EPO}$ therapy

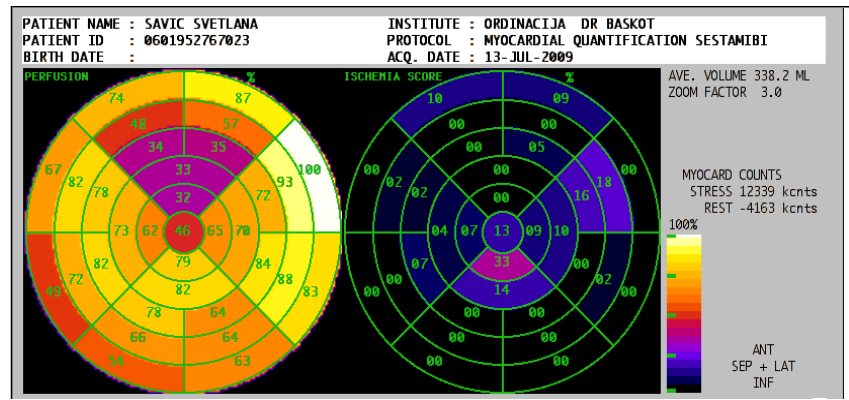

quantification of perfusion by Stierner - segments quantification 07.13.2009

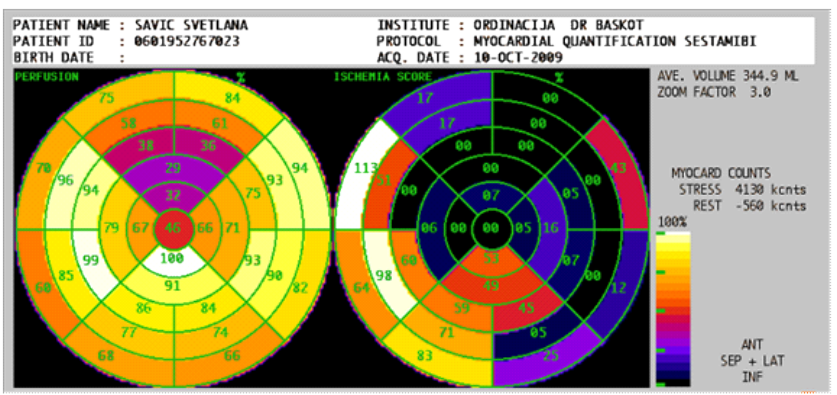

quantification of perfusion by Stierner - segments quantification 10/10/2009 


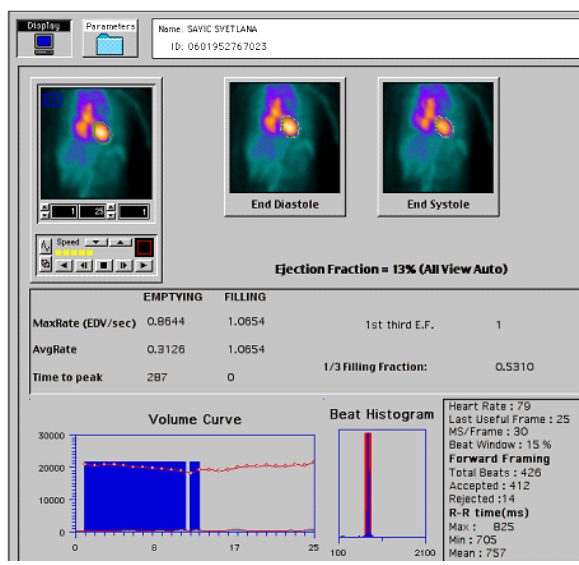

radionuclide ventriculography (RNV) performed 07.17. 2009 before therapy

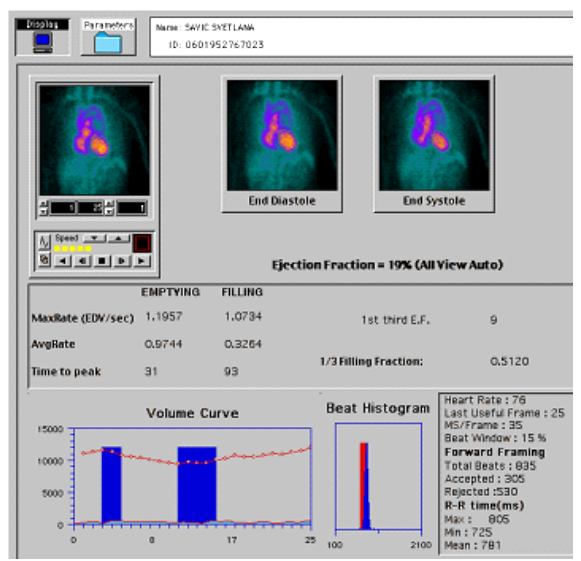

radionuclide ventriculography $(\mathrm{RNV})$ performed 10.12.2009 after therapy

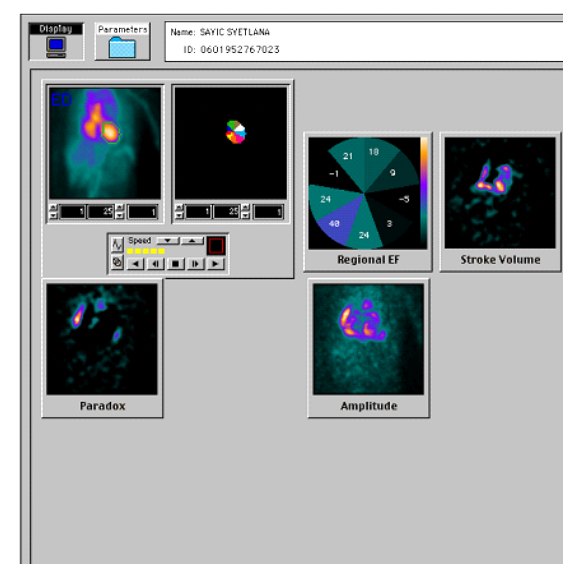

radionuclide ventriculography (RNV) regional ejection fraction

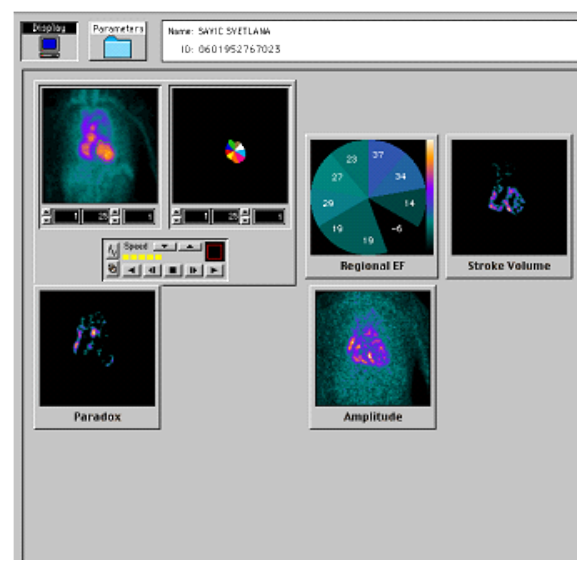

radionuclide ventriculography (RNV) regional ejection fraction 
Case report 2. Male with non-ischemic heart failure

SPECT scan performed before $\mathrm{HBO}=\mathrm{EPO}$ therapy

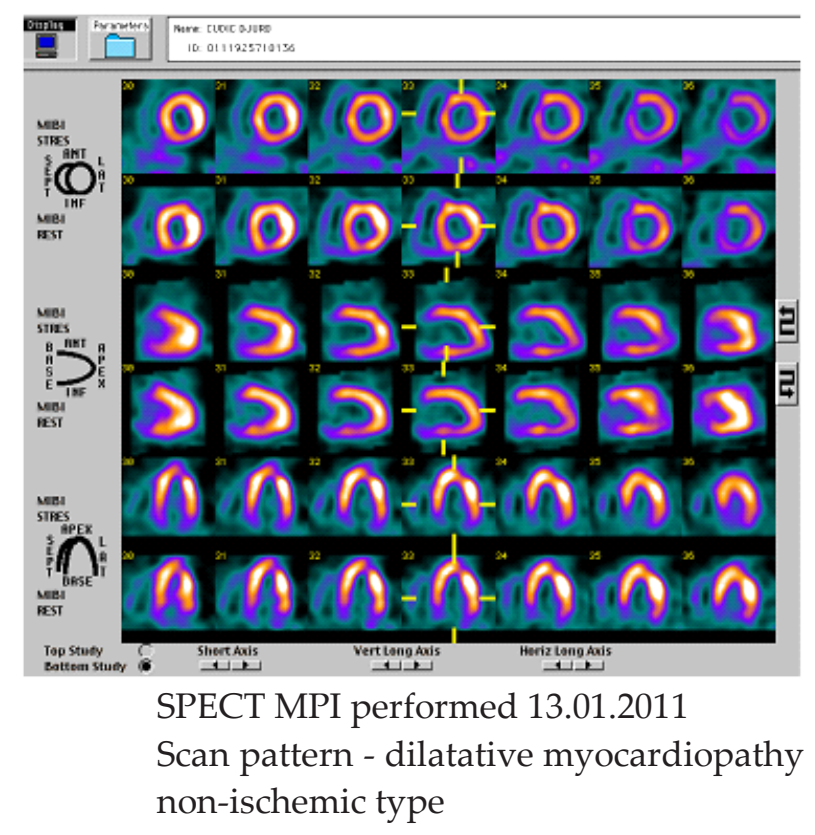

Radionuclide venticulography performed before and after $\mathrm{HBO}=\mathrm{EPO}$ therapy when we seen the greatest increase of LVEF with improve wall motion and regional kinetics 

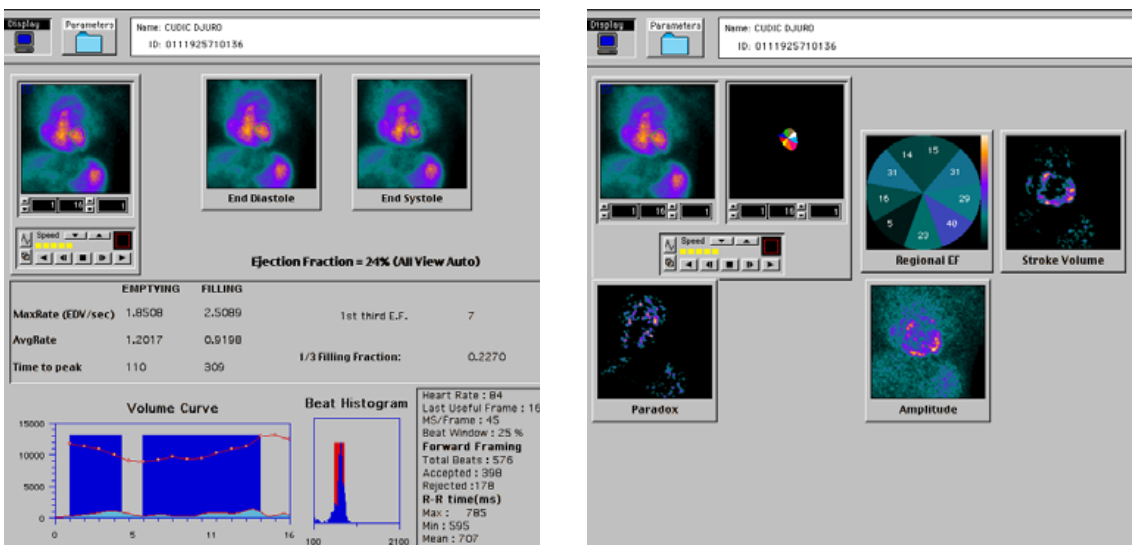

RNV with global and regional ejection fraction performed 01.17. 2011 - before therapy
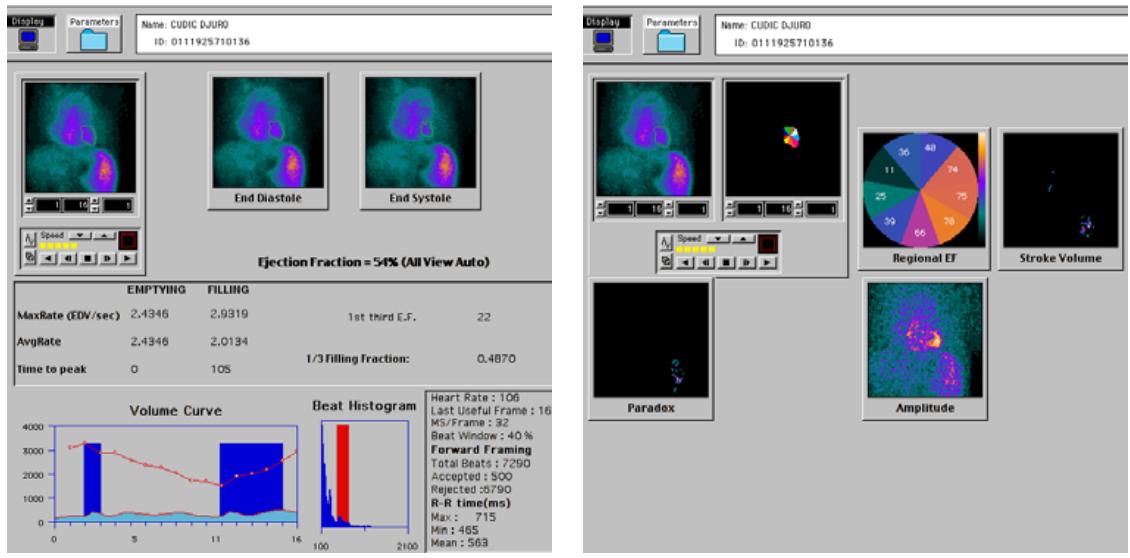

RNV with global and regional ejection fraction performed 03.23. 2011 - after therapy

\section{Summary}

The field of cardiovascular imaging is changing. In one hand, myocardial perfusion imaging is a well/established clinical technique for the diagnostic and prognostic workup of coronary artery disease. It has been the mainstay of nuclear cardiology for decades. On the other hand, several alternative imaging methodologies for noninvasive functional assessment of ischemic heart disease have emerged, and noninvasive coronary angiography is becoming a clinical reality. Nuclear imaging technology has progressed significantly toward higher sensitivity and resolution, and novel, highly specific radiotracers have been introduced. These developments are indicator of steady evolution of nuclear cardiology beyond the assessment of myocardial perfusion and toward characterization of biologic events on the tissue level. It is 
hoped that radiotracers techniques, with their unique translational potential and their superior detection sensitivity, will take a leading role in personalized cardiovascular medicine, in which therapeutic and/or preventive strategies are based on individual disease biology. The value of more specific imaging targets, which are increasingly entering clinical practice. This includes imaging of heart failure, absolute quantification of myocardial blood flow, imaging of myocardial metabolism, and imaging of the cardiac autonomic nervous system.

The goal of this chapter is to provide the reader with a comprehensive overview of the most recent development in nuclear cardiology, in the era of interventional cardiology. It is hoped that the reader, after going through this article, will share the enthusiasm of the author for this discipline, which holds the potential to be a key component in the new paradigm of early detection coronary artery disease for indication for interventional cardiology, as well as assessment new therapeutic effect $(\mathrm{HBO}+\mathrm{EPO})$ of heart failure.

\section{Author details}

Branislav Baskot ${ }^{1}$, Igor Ivanov ${ }^{2}$, Dragan Kovacevic ${ }^{2}$, Slobodan Obradovic ${ }^{3}$,

Nenad Ratkovic ${ }^{3}$ and Miodrag Zivkovic ${ }^{4}$

1 Private “Clinic Dr Baskot”, Belgrade, Serbia

2 Institute for Cardiovascular Disease Sremska Kamenica, Serbia

3 Clinic for Urgent Medicine, Medical Military Academy, Belgrade, Serbia

4 HBO Medical Center, Medical Practice for Hyperbaric Oxygenation Therapy, Belgrade, Serbia

\section{References}

[1] ACC/AHA/ASNC Guidelines for the Clinical Use of Cardiac Radionuclide Imaging. A Report of the American College of Cardiology/American Heart Association Task Force on Practice Guidelines (ACC/AHA/ASNC Committee to Revise the 1995 Guidelines for the Clinical Use of cardiac Radionuclide Imaging). Journal of the American Coll Cardiol October. 1. 2003. ACC/AHA/ASNC Practice Guidelines 01-69.

[2] Branislav Baskot: Nuclear cardiology - determination of culprit lesion. Belgrade: Andrejevic foundation; 2006.

[3] Garry V. Heller, Robert C. Hendel: Nuclear Cardiology - practical application 259-71 The McGroaw-Hill Companies, Inc. 2004 
[4] Masud H. Khandaker, Tod D. Miller, Panithaya Chateronthaitawee, J. Wells Askew, David O. Hodge, Raymond J. Gibbons: Stress single photon emission computed tomography for detection of coronary artery disease and risk stratification of asymptomatic patients at moderate risk. Journal of Nuclear Cardiology Vol 16, No 4;516-23 July/August 2009

[5] Shaw LJ, Hendel R., Borges-Neto S. Lauer MS: Prognostic value of normal exercise and adenosine $(99 \mathrm{~m})$ Tc-tetrofosmine SPECT imaging; results from the multicenter registry of 4,728 patients. J Nucl Med 44: 134, 2003

[6] Baskot B, Rafajlovski S, Ristić-Angelkov A, Obradović S, Gligić B, Orozović V, Agbaba N.: Study of efficacy and safety of pharmacological stress tests in nuclear cardiology. Vojnosanit Pregl. 2009 Mar;66(3):193-8. Serbian.

[7] Michael I. Miyamoto, Sharon L. Vernicoto, Haresh Majmundar, Gregory S. Thomas: Pharmacological stress myocardial perfusion imaging: A practical approach. Journal of Nuclear Cardiology 2007; vol 14 No 2, 250-55

[8] Georg A. Beller: Compliance with appropriate use criteria for cardiac radionuclide imaging. Journal of Nuclear Cardiology vol 17; No 2;165-67 March/April 2010

[9] Tim J.F., Johannes C. Kelder, Herbert W.M. Plokker, J. Fred Verzijlbergen, Norbert M. van Hemel: Myocardial perfusion SPECT identifies patients with left bundle branch block patterns at high risk for future cardiac events. Journal of Nuclear Cardiology vol 17; No 2;216-24 March/April 2010

[10] Georg A. Beller; Implications of randomized studies of medical therapy vs revascularization for reducing rising costs of helth care. Journal of Nuclear Cradiology vol 16. No 4;483-85 July/August 2009

[11] American Heart Association Writing Group on Myocardial Segmentation and Registration for Cardiac Imaging. Standardized myocardial segmentation and nomenclature for tomographic imaging of the heart: A statement for healthcare professionals from the Cardiac Imaging Committee of the Council on Clinical Cardiology of the American Heart Association. Circulation 2002; 105:539-42.

[12] Gary V. Heller, Robert C. Hendel.: Nuclear Cardiology Practical Applications. McGraw-Hill medical Publishing divison. The McGraw-Hill Companies, Inc. Copyright 2004; 193-243

[13] Udelson JE., Beshansky JR., Ballin DS.: Myocardial perfusion imaging for evaluation and triage of patients with suspected acute cardiac ischemia: a randomized controlled trial. JAMA 2002; 288:2693-2700

[14] Baskot B., Jankovic Z., Obradovic S., Rusovic S., Orozovic V., Gligic B., Jung R., Ivanovic V., Pavlovic M., Ratkovic N.,: Diagnostic significance of myocardial perfusion scintigraphy in identification and localization of culprit lesions in patients undergoing elective PTCA. VSP vol 65; No 2 (158-62) ; 2008 
[15] Leslee J Shaw, Allen Taylor, Paolo Raggi, Daniel S Berman: Role of noninvasive imaging in asymptomatic high/risk patients. J Nucl Cardiol 2006; vol 13 No2(156-62).

[16] AN Clarc, GA Beller: The present role of nuclear cardiology in clinical practice. The quarterly journal of Nuclear Medicine and Molecular Imaging. vol. 49 No 1(43-58) March 2005.

[17] Barry L. Zaret, George A. Beller: Clinical Nuclear Cardiology; state of the art and future directions. Elsevier Mosby. 2005.

[18] Vasken Dilsizian, Jagat Narula; Atlas of Nuclear Cardiology - second edition. Current medicine LLC 2006.

[19] Baskot B, Zivković M, Tepić S, Obradović S.: Evaluation of the therapeutic effect of hyperbaric oxygenation and erithropoietin in the treatment of chronic heart failure using myocardial perfusion scintigraphy G/SPECT. Vojnosanit Pregl. 2009 May;66(5): 399-402.

[20] Zivkovic Miodrag: Guide for Hyperbaric Medicine. Serbian Health Organization, Belgrade, Serbia. - 2010.

[21] Zivkovic M., Todorovic V., Tepic S., Jakovljevic V. Synergistic application of hyperbaric oxygenation therapy and erythropoietin in treatment of ch ronic heart failure. Medical review, No. 1-2, pp. 19/24. Novi Sad Serbia, 2007 